\title{
Advances in dengue vaccine: a review study
}

\begin{abstract}
Dengue is now a global burden with about 3.97 billion people living in 128 countries at risk and 400 million infections occurring every year. Four closely related serotypes have been implicated in the global burden: DENV 1-4. It has been reported that there are serotype specific differences in clinical manifestations. This systematic review is based on PRISMA guidelines. It discusses two types of DENV vaccine based on inclusion criteria: Tetravalent dengue vaccine (TDV) and dengue DNA vaccine that are in clinical studies. A number of studies evaluated the safety, reactogenicity, and immunogenicity of TDV especially Sanofi Pasteur's CYD-TDV. Most of the studies reported that the vaccine candidate is safe and elicit efficient neutralizing antibody. As part of the global effort to develop DENV vaccine, the US National Institute of Health also initiated a program which culminated in the development of a monovalent dengue vaccine candidate called rDEN4 $\Delta 30$. Five different formulation were evaluated in clinical studies. Only a paper described a randomized controlled study of DENV DNA vaccine. It reported that the vaccine candidate is safe at the given dose. Studies are needed to evaluate the immunological profile of dengue vaccine in children and those in endemic areas. The future of DENV vaccine effort looks bright and it is hoped that soon an efficient DENV vaccine will be available for human use.
\end{abstract}

Volume 4 Issue 3 - 2016

\author{
AbubakarYaro \\ Department of Clinical Research, AHRO Institute of Health \& \\ Research, Ghana \\ Correspondence: Abubakar Yaro, Department of Clinical \\ Research, AHRO Institute of Health \& Research, Dr. \\ Yaro Laboratory, Ghana, PO Box CT 896I, Cantonments Accra \\ Ghana, Ghana, Email abubakar_yarogh@yhoo.com
}

Received: November 22, 2016 | Published: December 30, 2016

Keywords: Dengue virus, Dengue fever, Vaccine, DNA vaccine, Vaccine protection, Antibodies, T-cells, TDV

Abbreviations: TDV, Tetravalent Dengue Vaccine; DENV, Dengue Virus; DSS, Dengue Shock Syndrome; DHF, Dengue Hemorrhagic Fever; DDV, DNA Vaccine

\section{Introduction}

Dengue virus (DENV) is a vector-borne viral disease that cause more human morbidity and mortality worldwide than any other arthropod-borne virus. It is estimated that 3.97 billion people living in 128 countries are at risk of dengue, 400 million infections annually, and 96 million asymptomatic cases occurring every year. ${ }^{1-4}$ Four closely related dengue serotypes cause the disease: DENV-1 to -4 . Serotype specific differences in clinical manifestations have been reported In a cross-sectional study, Balmaseda et al. ${ }^{5}$ reported that among hospitalized children in Nicaragua over 3 years period DENV-2 was associated with more shock and internal hemorrhage while DENV1 was associated with increased vascular permeability. Furthermore, DENV-1 was associated with more hospitalized primary dengue cases and more primary DENV infection with severe manifestations. Others reported that DENV-2 and DENV-3 may cause more severe diseases than the other serotypes and that DENV-4 is associated with milder illness. ${ }^{6-12}$ Certain genotypes within particular serotypes are associated with epidemics of dengue hemorrhagic fever (DHF) vs. classic dengue. ${ }^{11,13}$ Aedes aegypt mosquitoes are the primary vectors of DENV throughout the tropics. ${ }^{14} \mathrm{DENV}$ infection is categorized as asymptomatic infection, subclinical infection, undifferentiated fever, dengue fever, DHF with or without dengue shock syndrome (DSS) and other severe forms of dengue. DHF is characterized by fever, bleeding diathesis, and a tendency to develop a potentially fatal shock syndrome. Clinical manifestations of dengue infection range from mild dengue fever to severe plasma leakage with hemorrhagic manifestations. Thrombocytopenia is an important finding in patients with dengue. A study reported that platelet count of dengue patients can be normal or abnormally high or low. This should be used for future diagnosis ${ }^{15-17}$ Management of dengue patient is to have good fluid replacement therapy until recovery of platelet. Multiple factors have been suggested to contribute to severe dengue such as secondary infections, age, viral load and infecting serotype and genotype. ${ }^{6-8}$
Dengue infection is spreading at an alarming rate around the globe and statistics shows that the disease has increased dramatically since 1980, with epidemics occurring in both eastern and western Africa. ${ }^{18,19}$ The WHO statistics indicates that $2.4 \%$ of the global burden of dengue hemorrhagic fever is in Africa and one-fifth of the populations are at risk. $^{17}$

Currently there is no specific antiviral agents and vaccine for treating and/ or preventing dengue infection., ${ }^{2,4}$ The prevention technique is based on control of Ae aegypt which is failing in most countries as a result of lack of resources and apathy. Taking into consideration the global burden of dengue, the best preventive intervention is the use of vaccines. Studies has shown that longterm homotypic immunity is generated after infection with a single DENV serotype and pre-existing immunity to one DENV serotype is a risk factor for severe disease upon secondary heterotypic infection. Based on these, a potential DENV vaccine must induce long-lasting protective immunity against all four DENV serotypes. ${ }^{20-25}$ The aim of this study is to summarize current data available on dengue vaccines and provide an insight into the safety-efficacy profile and the potential drawbacks of dengue vaccines based on safety-efficacy studies.

\section{Method of data collection}

A systematic review based on PRISMA guidelines. ${ }^{20}$ was performed. The search was conducted across the following electronic databases: PubMed, Embase, WHO Library information, and Google scholar using different combinations of search terms (with synonyms and closely related words) such as: "dengue virus", "dengue vaccine", "randomized controlled trials" "clinical study", "efficacy", and "effectiveness". The titles and abstract of all articles were screened to determine their eligibility. Other articles that showed up as related during the search were screened. The full text of eligible articles were retrieved and further assessed for inclusion. Conferences proceedings and agency reports were searched to identify ongoing or unpublished. Authors were contacted to share preliminary summaries or unpublished studies to assess their eligibility for inclusion in the review. Searches were limited to between January 2010 and 
January 2016, and limited to English language. Eligible studies were randomized controlled trials that assessed the efficacy of a dengue vaccine and possess clinical trial registration number.

A total of 29,188 articles were identified by searching PubMed (255), Embase (280), WHO library of information (453), and Google scholar search $(28,200)$ respectively. The identified studies were screened on the basis of original study and its relevance to the aim of this review. Studies that did not meet the inclusion criteria were excluded.

Of the 29,188 screened reports, 11 articles were finally selected on the basis of inclusion criteria to describe tetravalent Dengue vaccine (TDV) and Dengue DNA vaccine (DDV). Additional studies were obtained through citation tracking of review and original articles.

Tetravalent Dengue Vaccine (TDV): A live attenuated, tetravalent DENV vaccine candidate was generated using reverse genetics which is able to provide immunity to each of the four serogroup of DENV. Attenuation was achieved by generating recombinant DENV which are modified by deletion, or alteration, by antigenic chimerization between two related DENV using the following two strategies: 1 . Introduction of an attenuating 30 nucleotide deletion (Delta 30) mutant into the 3' untranslated region of DENV-1 and DENV-4, and 2. Replacement of structural proteins of the attenuated rDENV Delta 30 vaccine candidate with those of DENV2 or DENV3 Attenuation of the four monovalent vaccine candidate was achieved for rhesus monkeys or humans. One of the candidate that is showing promise is recombinant, live-attenuated tetravalent dengue disease vaccine (CYD-TDV) produced by Sanofi-Pasteur, Lyon France. CYD-TDV is in the late stage of clinical development and has been evaluated in different populations and age groups. It is made of four recombinant (CYD-1 to -4) which expresses the dengue pre-membrane and envelope proteins of one of the four DENV serotypes and the nonstructural as well as capsid protein of an attenuated yellow fever (YF) vaccine in YF-17D (Figure 1). ${ }^{26,27}$. Hadinegoro et al assessed CYDTDV in two phases three randomized efficacy trials involving 21000 children between the ages of 2 and 14 year in the Asia-Pacific region and between the ages of 9 and 16 years in Latin America. The vaccine was administered in three doses at baseline, 6 months, and 12 months. Vaccine efficacy against virologically confirmed dengue while safety was assessed during a 25 months efficacy surveillance phase. Reactogenicity and immunogenicity were also assessed in a subgroup of participants. They reported that the vaccination significantly reduced the incidence of virologically confirmed dengue and showed acceptable and safer reactogenicity profiles.$^{28}$ In another randomized, placebo-controlled study, Durbina et al. ${ }^{29}$ evaluated the safety and immunogenicity of four different tetravalent dengue vaccines was evaluated in 113 flavivirus-naïve adults. The serum neutralizing antibody levels to all four dengue vaccines were measured on days $0,28,48$, and 180. A single dose of the vaccine was administered resulted in induction of trivalent or better neutralizing antibody response in $75 \%-90 \%$ of vaccines. It was also found that trivalent or better response correlated with rash and with non-black race. Black race was significantly associated with reduced incidence of vaccine viremia. ${ }^{29}$ This reflects the effect of race on disease severity in natural dengue infection.

In a randomized controlled phase I study in Philippine, Capeding et al. ${ }^{30}$ reported that a vaccine regime of either 3 TDV vaccination administered over a year or two TDV vaccination given more than 8 months apart led to a balanced antibody response to all four dengue serotypes among flavivirus-exposed populace that included children. ${ }^{31}$ The reactogenicity did not increase with successive vaccination and was not higher in children than in adults and adolescents. Others have also reported of the efficacy of TDV in clinical studies. ${ }^{32-36}$ The safety profiles of this vaccine have been evaluated. The study by Hadinegoro et al reported that the vaccine was safe in all age groups in the first year of the study but during the third year, higher cases of hospitalizations were reported in the vaccinated group in comparison to the unvaccinated group for those individuals below the age of 9 . There was no report of increased risk among subjects aged 9 and above with a reported more stable and sustained hospitalization reduction into the third year although reduction in efficacy was reported from the first to third year. Leo et al reported that in children aged 9 and above, efficacy against virologically confirmed dengue of any severity was $64 \%$ while against hospitalization $81 \%$ as well as severe disease $93 \%$. Efficacy was seen to decline over the years which could be attributed to waning vaccine efficacy or increase in enhanced disease reported among the seronegatives during the third year. Due to the reported higher efficacy and lack of any safety signal, the best age groups to benefit from this vaccine are individuals from the age of 9 to 1635 . There is data on safety and immunogenicity for individuals aged up to 45 but there is no efficacy data for individuals above the age of $1636,{ }^{37}$ Osorio et al. ${ }^{31}$ reported that there is acceptable tolerability and immunogenicity of the tetravalent DENVAX formulation in healthy, flavivirus-naïve adults but more clinical studies are needed to test DENVAX in different age groups in DENV-endemic areas. Although TDV is safe, a number of adverse events have been reported. Sirivichayakal et al. ${ }^{34}$ reported of the following solicited adverse events (AEs): injection site pain, itching and erythema which was mostly mild while Rupp et al reported of the following unsolicited AEs: headache, fatigue, myalgia and injection site pain. ${ }^{38}$

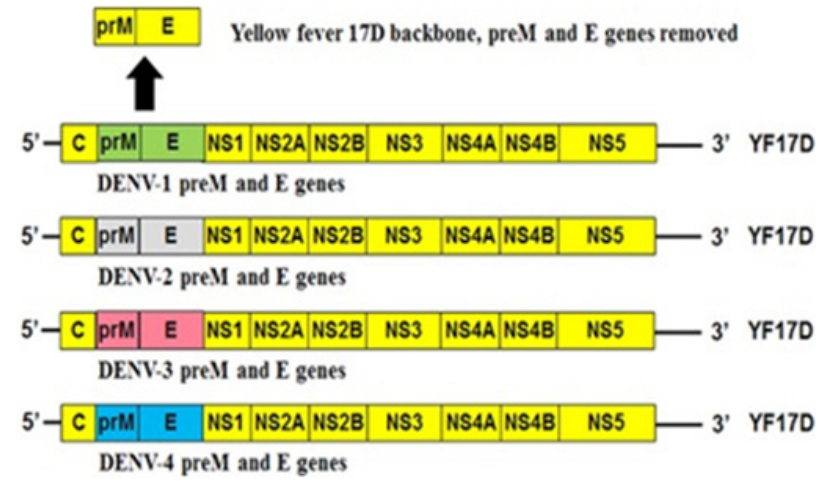

Figure I Structural representation of CYD-TDV (Source:Thomas, 20I4).

The U.S. National Institute of Health $(\mathrm{NIH})$ as part of the global effort to develop a DENV vaccine initiated a program with the aim of developing a live attenuated tetravalent dengue vaccine which could induce protection against all four DENV serotypes. ${ }^{39}$ Recombinant technology was used in two primary attenuation strategies: deletions in the 3' untranslated region and structural gene chimerization. A prototype monovalent candidate vaccine termed rDEN4 $\triangle 30$ that contains all the structural and non-structural proteins of wild type DENV-4 was developed. It was attenuated at 3'UTR. When the analogues 30 nucleotide was removed, it resulted in the creation of additional vaccine candidate. Furthermore, chimeric vaccine candidate were developed by the substitution of prM and $\mathrm{E}$ genes from each of the rDEN $4 \Delta 30$ vaccine candidates. Five of these formulations, designated as TV001-TV005 (Figure 2) were evaluated in clinical studies. ${ }^{29,41}$

During the clinical studies, a very important observation was made: chimerization of the viruses was highly attenuating and even in 
some cases over-attenuating. The first DENV-3 vaccine candidate that was analyzed was a chimeric virus construction which had DENV-3 and rDEN $4 \Delta 30$ background. The strategy was similar in the SanofiPasteur vaccine but the prM and E of DENV-3 was substituted with another DENV virus rather than the YF 17D virus. Upon evaluation in human, the developed virus, $\mathrm{rDEN} 3 / 4 \Delta 30$ was found to be poorly infectious because it induced seroconversion to DENV-3 in $20 \%$ of the subjects. ${ }^{40}$ The failure of $\mathrm{rDEN} 3 / 4 \Delta 30$ led to the evaluation of two other DENV-3 vaccine candidates: $\operatorname{rDEN} 3 \Delta 30 / 31$ and rDEN3$3^{\prime} \mathrm{D} 4 \Delta 30$. The first vaccine candidate includes 31 nucleotide deletion in the 3' UTR plus addition to the 30 nucleotide deletion homologues to that found in $\operatorname{rDEN} 1 \Delta 30$ and $\operatorname{rDEN} 4 \Delta 30$. The latter is a chimeric virus in which the entire 3' UTR of rDEN4 $\triangle 30$ replacing that of DENV-3. It was found that both viruses induced seroconversion to DENV-3 in more than $80 \%$ of subjects when evaluated as monovalent viruses. The latter candidate appeared to be less infectious than the former. This resulted in $\mathrm{rDEN} 3 \Delta 30 / 31$ been chosen as the DENV-3 component of the tetravalent vaccine.

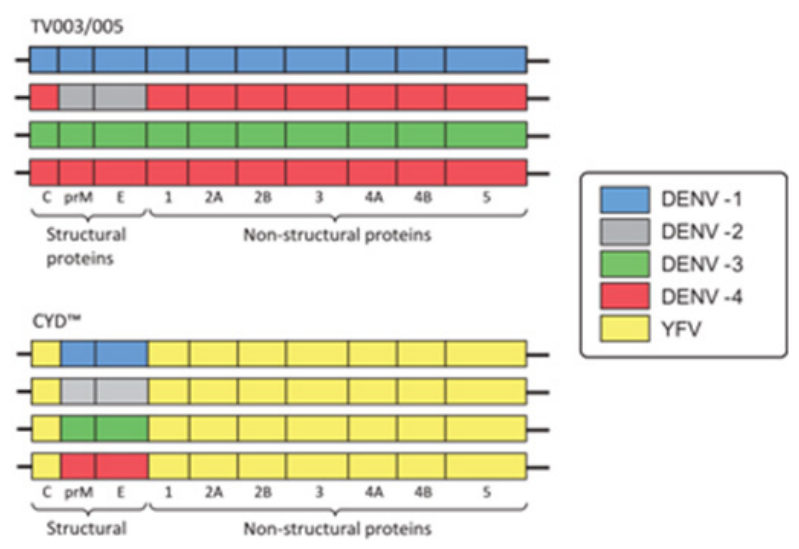

Figure 2 Structural representative of TV003/005 (Source:Whitehead, 2016).

Dengue DNA Vaccine (DDV): Only one paper carried randomized controlled study on Dengue DNA vaccine. In a clinical study by Beckett et al. ${ }^{41}$ a candidate Dengue DNA vaccine was construct was developed through the incorporation of pre-membrane and enveloped genes into a plasmid vector. Their phase 1 clinical trial was conducted using the DENV-1 vaccine construct (DIME 100) which involved 22 healthy flavivirus-naïve adults who were assigned to one of two groups. Each of the group was given three intramuscular injections at 0,1 , and 5 months of either high dose of $5.0 \mathrm{mg}$ or low dose of $1.0 \mathrm{mg}$ DNA vaccine utilizing the needle-free Biojector ${ }^{\circledR} 2000$. They reported of a number of solicited signs and symptoms which included local mild pain or tenderness, local swelling, muscle pain, and fatigue. Five subjects in the high dose group and none in the low dose group developed detectable anti-dengue neutralizing antibodies. T-cell IFN gamma responses were found in $50 \%$ and $83.3 \%$ of subjects in the low and high dose groups respectively. This means the safety profile of the vaccine candidate is acceptable at both doses. The authors therefore concluded that the result of the study highlights the safety profile and favorable reactogenicity of a DENV-1 DNA vaccine.

\section{Conclusion}

There is an urgent need for an efficacious and affordable tetravalent DENV vaccine. However, lack of adequate animal disease model and identifying immune correlation of disease protection are some of the major obstacles impeding the development of a successful DENV vaccine. The Sanofi Pasteur CYD has been licensed in some few countries but it recorded poor efficacy in dengue naïve individuals during phase III clinical study. This could be attributed to the yellow fever virus backbones which lack the essential dengue $\mathrm{T}$ cell epitopes of the nonstructural region which have been shown to play important role in providing protection against dengue. ${ }^{42,43}$ Other studies also implicated immunity to dengue NS1 as essential in providing protection which CYD-TDV lacks. It has also been reported that it generated lots of cross-reactive nonneutralzing/ enhancing antibodies. It has therefore been proposed that it is not only the presence of dengue neutralizing antibodies that may hold the key to a successful dengue vaccine candidate but also DENV serotype-specific neutralizing antibodies. ${ }^{44,45}$ Therefore an effective dengue vaccine must be designed with the capability of inducing predominantly serotypespecific neutralizing antibodies (protective) in the absence of serotype cross-reactive neutralizing antibodies (disease enhancing). ${ }^{46}$ Studies are recommended to elucidate the immunological profile of dengue vaccine in children and those in endemic areas. The list of potential dengue vaccine is growing and despite the low efficacy recorded so far, it is hoped that soon an efficacious dengue vaccine will be available.

\section{Acknowledgments}

None.

\section{Conflicts of interest}

None.

\section{References}

1. Bhatt S, Gething PW, Brady OJ, et al. The global distribution and burden of dengue. Nature. 2013;496(7446):504-507.

2. Special Programme for research and training in tropical diseases, World Health Organization. Dengue: Guidelines for diagnosis, treatment, prevention, and control; Geneva: TDR: World Health Organization, USA. 2009;p. 147.

3. Brady OJ, Gething PW, Bhatt S, et al. Refining the global spatial limits of dengue virus transmission by evidence-based consensus. PLoS Negl Trop Dis. 2012;6(8):e1760.

4. Lambrechts L, Scott TW, Gubler DJ. Consequences of the expanding global distribution of Aedes albopictus for dengue virus transmission. PLoS Negl Trop Dis. 2010;4(5):e646.

5. Balmaseda A, Hammond SN, Pérez L, et al. Serotype-specific differences in clinical manifestations of dengue. Am J Trop Med Hyg. 2006;74(3):449-456.

6. Burke DS, Nisalak A, Johnson DE, et al. A prospective study of dengue infections in Bangkok. Am J Trop Med Hyg. 1988;38(1):172-180.

7. Guzman MG, Kouri G. Dengue and dengue hemorrhagic fever in the Americas: lessons and challenges. J Clin Virol. 2003;27(1):1-13.

8. Rico-Hesse R, Harrison LM, Salas RA, et al. Origins of dengue type 2 viruses associated with increased pathogenicity in the Americas. Virology. 1997;230(2):244-251.

9. Sangkawibha N, Rojanasuphot S, Ahandrik S, et al. Risk factors in dengue shock syndrome: a prospective epidemiologic study in Rayong, Thailand. I. The 1980 outbreak. Am J Epidemiol. 1984;120(5):653-669.

10. Nisalak A, Endy TP, Nimmannitya S, et al. Serotype-specific dengue virus circulation and dengue disease in Bangkok, Thailand from 1973-1979. Am J Trop Med Hyg. 2003;68(2):191-202.

11. Messer WB, Gubler DJ, Harris E, Sivananthan K, de Silva AM, et al. (2003) Emergence and global spread of a dengue serotype 3, subtype III virus. Emerg Infect Dis 9(7): 800-809.

12. Vaughn DW, Green S, Kalayanarooj S, Innis BL, Nimmannitya S, et al. (2000) Dengue viremia titer, antibody response pattern, and virus serotype correlates with disease severity. J Infect Dis 181(1): 2-9. 
13. Rico-Hesse R, Harrison LM, Salas RA, Tovar D, Nisalak A, et al. (2003) Origins of dengue type 2 viruses associated with increased pathogenicity in the Americas. Virology 230(2): 244-251.

14. Mboera LE, Mweya CN, Rumisha SF, Tungu PK, Stanley G, et al. (2016) The risk of dengue virus transmission in Dar es Salaam, Tanzania during an epidemic period of 2014. PLoS Negl Trop Dis 10(1): e0004313.

15. Amarasinghe A, Kuritsk JN, Letson GW, Margolis HS (2011) Dengue virus infection in Africa. Emerg Infect Dis 17(8): 1349-1354.

16. Sang RC (2007) Dengue in Africa In: Report of the scientific working group meeting on dengue: Geneva. WHO Special Programme for Research and Training in Tropical Dieases, USA, p. 50-52.

17. LeDuc JW, Esteves K, (2004) Dengue and dengue hemorrhagic fever In: Murray CJ, et al. (Eds,). The global epidemiology of infectious diseases. Volume IV Global burden of disease and injury series Geneva: World Health Organization, USA, pp. 219-242.

18. Wiwanitkit V (2010) Dengue fever: diagnosis and treatment. Expert Rev Anti Infect Ther 8(7): 841-845.

19. Wiwanitkit V, Manusvanich P (2004) Can hematocrit and platelet determination on admission predict shock in hospitalized children with dengue hemorrhagic fever? A clinical observation from a small outbreak, Clin Appl Thromb Hemost 10(1): 65-67.

20. Moher D, Liberati A, Tetzlaff J, Altman DG (2009) Preferred reporting items for systematic reviews and meta-analysis: The Prisma statement. BMJ 339: b2535.

21. Sabin A (1953) Research on dengue during World War II. Am J Trop Med Hyg 1(1): 30-50.

22. Okuno Y, Fukunaga T, Tadano M, Fukai K, Ikeda T, et al. (1943) Serological studies on volunteers inoculated experimentally with a dengue virus strain in 1943. Biken J 26(4): 161-163.

23. Papaevangelou G, Halstead SB (1977) Infection with two dengue viruses in Greece in the 20th century. Did dengue hemorrhagic fever occur in the 1928 epidemic? Am J Trop Med Hyg 80(3): 46-51.

24. Burke DS, Nisalak A, Johnson DE, Scott RM (1988) A prospective study of dengue infections in Bangkok. Am J Trop Med Hyg 38(1): 172-180.

25. Kliks SC, Nisalak A, Brandt WE, Wahl L, Burke DS (1989) Antibodydependent enhancement of dengue virus growth in human monocytes as a risk factor for dengue hemorrhagic fever. Am J Trop Med Hgy 40(4): 444-451.

26. Blaney JE, Durbin AP, Murphy BR, Whitehead SS (2006) Development of a live attenuated dengue virus vaccine using reverse genetics. Virol Immunol 19(1): 10-32.

27. Thomas SJ (2014) Developing a dengue vaccine: Progress and future challenges. Ann NY Acad Sci 1323: 140-150.

28. Hadinegoro SR, Arredondo-García JL, Capeding MR, Deseda C, Chotpitayasunondh T, et al. (2015) Efficacy and long-term safety of a Dengue vaccine in regions of endemic disease. NEJM 375(13): 1195-1206.

29. Durbin AP, Kirkpatrick BD, Pierce KK, Elwood D, Larsson CJ, et al. (2013) A single dose of any four different live attenuated tetravalent Dengue vaccine is safe and immunogenic in Flavivirus-naïve adults: A randomized, double-blind clinical trial. J Infect Dis 207(6): 957-965.

30. Capeding RZ, Luna IA, Bomasang E, Lupisan S, Lang J, et al. (2011) Live-attenuated, tetravalent dengue vaccine in children, adolescents, and adults in a dengue endemic country: Randomized controlled phase I trial in Philippine. Vaccines 29(22): 3863-3872.

31. Osorio JE, Velez ID, Thomson C, Lopez L, Jimenez A, et al. (2014) Safety and immunogenicity of a recombination live attenuated tetravalent dengue vaccine (DENVAX) in flavivirus-naïve healthy adults in Colombia: a randomized, placebo-controlled, phase 1 study. Lancet Infect Dis 14(9): 830-838.

32. Lanata CF, Andrade T, Gil AI, Terrones C, Valladolid O, et al. (2012) Immunogenicity and safety of tetravalent dengue vaccine in 2-11 years olds previously vaccinated against yellow fever: randomized, controlled, phase II study in Piura, Peru. Vaccine 30(41): 5935-5941.

33. George SL, Wong MA, Dube TJ, Boroughs KL, Stovall JL, et al. (2015) Safety and immunogenicity of a live-attenuated tetravalent dengue vaccine candidate in flavivirus-naïve adults: a randomized, doubleblinded phase I clinical trial. J Infect Dis 212(7): 1032-1041.

34. Sirivichayakul C, Barranco-Santana EA, Esquilin-Rivera I, Oh HM, Raanan M, et al. (2016) Safety and immunogenicity of a tetravalent dengue vaccine candidate in healthy children and adults in Dengueendemic regions: A randomized, placebo-controlled phase 2 study. J Infect Dis 213(10): 1562-1572.

35. Leo YS, Wilder-Smith A, Archuleta S, Shek LP, Chong CY, et al. (2012) Immunogenicity and safety of recombination tetravalent dengue vaccine (CYD-TDV) in individuals aged 2-45 years: Phase II randomized controlled trial in Singapore. Human Vaccines \& Immunotherapy 8(9): 1259-1271.

36. Lanata CF, Andrade T, Gil AI, Terrones C, Valladolid O, et al. (2012) Immunogenicity and safety of tetravalent dengue vaccine in 2-11 years olds previously vaccinated against yellow fever: randomized, controlled, phase II study in Piura, Peru. Vaccine 30(41): 5935-5941.

37. George SL, Wong MA, Dube TJ, Boroughs KL, Stovall JL, et al. (2015) Safety and immunogenicity of a live-attenuated tetravalent dengue vaccine candidate in flavivirus-naïve adults: a randomized, doubleblinded phase I clinical trial, J Infect Dis 212(7): 1032-1041.

38. Rupp R, Luckasen GJ, Kirstein JL, Osorio JE, Santangelo JD, et al (2015) Safety and immunogenicity of different dose and schedule of a live attenuated dengue vaccine (TDV) in healthy adults: A phase Ib randomized study. Vaccine 33(46): 6351-6359.

39. Whitehead SS (2016) Development of TV003/TV005, a single dose, highly immunogenic live attenuated dengue vaccine; what makes this vaccine different from the Sanofi-Pasteur CYDTM vaccine? Expert Review of Vaccines 15(4): 509-517.

40. Durbin AP, Kirkpatrick BD, Pierce KK, Schmidt AC, Whitehead SS (2011) Development and clinical evaluation of multiple investigational monovalent DENV vaccines to identify components for inclusion in a live attenuated tetravalent DEN vaccine. Vaccine 29(42): 7242-7250.

41. Beckett CG, Tjaden J, Burgess T, Danko JR, Tamminga C, et al. (2011): Evaluation of a prototype dengue-1 DNA vaccine in a phase 1 clinical trial. Vaccine 29(5): 960-968.

42. Weiskopf D, Angelo MA, de Azeredo EL, Sidney J, Greenbaum JA, et al. (2013) Comprehensive analysis of dengue virus-specific responses supports an HLA-linked protective role of CD8+ T cells. PNAS of USA 110(22): E2046-E2053.

43. Weiskopf D, Sette A (2014) T-cell immunity to infection with dengue virus in humans. Front immunol 5: 93

44. Modhiran N, Watterson D, Muller DA, Panetta AK, Sester DP, et al. (2015) Dengue virus NS1 activates cells via Toll-like receptor 4 and disrupts endothelial cell monolayer integrity. Sci Transl Med 7(304): 304ra142.

45. Beatty PR, Puerta-Guardo H, Killingbeck SS, Glasner DR, Hopkins K, et al. (2015) Dengue virus NS1 triggers endothelial permeability and vascular leak that is prevented by NS1 vaccination. Sci Transl Med 7(304): 304ra141.

46. Khetarpal N, Khanna I (2016) Dengue fever: causes, complications, and vaccine strategies. J of immunology research 2016: 6803098. 